\title{
Neuropelveological assessment of neuropathic pelvic pain
}

\author{
M. Possover • A. Forman
}

Received: 11 July 2013 / Accepted: 5 March 2014 / Published online: 21 March 2014

(C) Springer-Verlag Berlin Heidelberg 2014

\begin{abstract}
The aim of the present report is to emphasize the importance of taking neurological considerations into account in the diagnostic workup of chronic pelvic pain (CPP) of "unknown origin." Based on new knowledge of the functional neuroanatomy of the pelvis and recent developments in the treatment of pelvic neuropathies, we incorporated well-known neurologic diagnostic methods into the classical gynecological workup for CPP. "Neuropelveological" assessment of CPP in women requires a detailed gynecological and neurologic history, a classical gynecological workup, and an adapted "gynecological" examination of the pelvic nerves and plexuses. The present report provides guidelines for "neuropelveological" assessment of chronic pelvic pain in clinical practice. It emphasizes the benefits of taking "neurological" considerations into account when diagnosing chronic pelvic pain.
\end{abstract}

Keywords Pudendal neuralgia · Vulvodynia Coccygodynia Chronic pelvic pain $\cdot$ Genital pain · Neuropelveology

\section{Introduction}

Chronic pelvic pain (CPP) afflicts 7 to $24 \%$ of the population and is associated with impaired quality of life and high health care costs. Coccygeal, perianal, perineal, and/or genital pain are frequent complaints that usually occur as a result of common and easily recognizable organic disorders, such as anal fistulae, viral or bacterial infections, thrombosed hemorrhoids, genitoanal cancer, or other dermatologic pathologies. However, they can also

\footnotetext{
M. Possover $(\square)$

Center of Gynecologic Oncology \& Neuropelveology,

Possover International Medical Center,

Hirslanden Clinic, Zurich, Switzerland

e-mail: m.possover@possover.com

M. Possover · A. Forman

Dept of Gynecology \& Neuropelveology, University of Aarhus,

Aarhus, Denmark
}

occur under circumstances in which no organic cause can be identified. Chronic pelvic pain without any apparently etiology always constitutes a challenge for patients and clinicians. A correct diagnosis is crucial for etiologic treatment of the underlying cause. CPP can occur due to pelvic conditions that affect the nerves or to pathologies of the pelvic nerves and plexuses themselves. Therefore, knowledge beyond the limits of gynecology is required for proper diagnosis. Additional knowledge of pelvic neuroanatomy and clinical neurology is mandatory. This multidisciplinary field, referred to as neuropelveology, focuses on the diagnosis of pathologies and injuries to the pelvic nerves and plexuses responsible for the development of "non-gynecologic" chronic pelvic pain.

\section{Neuro-anatomical considerations}

The pelvis contains several parallel nerve systems. The most important nerve groups are the sacral plexus, the inferior hypogastric plexus, and the sensitive branches of the lumbar plexus (genitofemoral and ilioinguinal nerves). The sciatic and gluteal nerves as well as the pelvic splanchnic and pudendal nerves emerge from the sacral plexus.

The sacral plexus originates from the lumbosacral trunk (L4, L5, and S1) and the ventral roots of nerves S2 to S4. While most of the fibers originating from L5 and S1 running down into the sciatic nerve, sacral nerve roots $\mathrm{S} 3$ and $\mathrm{S} 4$ only innervate genito-anal areas without any fibers going to or from the leg. S2 is part of the pudendal nerve and sciatic nerve.

Sensory supply to the vulvar, perineal and perianal skin, and subcutaneous tissue of the lower two-thirds of the vagina is the pudendal nerve (PN). The pudendal nerve is a sensory and somatic nerve which originates from the ventral rami of the second to fourth (and occasionally fifth) sacral nerve roots. After branching from the sacral plexus, the nerve leaves the pelvis through the great sciatic notch, re-enters the pelvic cavity through the lesser sciatic notch, and finally travels to three main regions: the gluteal region, the pudendal canal, and the perineum. It accompanies the internal pudendal vessels 
upward and forward along the lateral wall of the ischiorectal fossa, being contained in a sheath of the obturator fascia termed the pudendal canal (Alcock's canal). The pudendal nerve gives off three distal branches - the inferior rectal nerve, the perineal nerve, and the dorsal nerve of the penis (in males) or the dorsal nerve of the clitoris (in females).

The pudendal nerve innervates not only the external genitalia of both sexes, but also the sphincters of the urinary bladder and rectum. As the bladder fills, the pudendal nerve becomes excited. Stimulation of the pudendal nerve results in contraction of the external urethral sphincter. Contraction of the external sphincter, coupled with that of the internal sphincter, maintains urethral pressure (resistance) higher than normal bladder pressure. The storage phase of the urinary bladder can be switched to the voiding phase either involuntarily (reflexively) or voluntarily. The pudendal nerve causes then relaxation of the levator ani so that the pelvic floor muscle relaxes. The pudendal nerve also signals the external sphincter to open. The sympathetic nerves send a message to the internal sphincter to relax and open, resulting in a lower urethral resistance. The PN is also known to have a potential modulatory effect on bladder function. Somatic afferent fibers of the pudendal nerve are supposed to project on sympathetic thoracolumbar neurons to the bladder neck and modulate their function. This neuromodulatory effect works exclusively at the spinal level and appears to be at least partly responsible for bladder neck competence and urinary continence [1]. Pudendal supply is not significant in the vaginal wall since there is no striated muscle, but efferent supply largely from the pudendal nerve controls the levator muscles that provide support, and influence function of the lower third of the vagina.

The pelvic visceral nerves arise chiefly from the hypogastric plexus (T1-L2). Visceral nerve supply is significant for the upper vagina, musculature, and glands. These nerves arise from the inferior hypogastric plexus, which gives rise to three other divisions. One division is the uterovaginal plexus (Frankenhausen's plexus) around the ureter and uterine artery. Fibers from the uterovaginal plexus accompany the vaginal artery and vein to the vagina. Parietal peritoneum in the pouch of Douglas is supplied by the visceral afferent nerves of the uterovaginal plexus. No parasympathetic fibers have been described in association with this hypogastric innervation of the vagina. The chief importance of vaginal parasympathetic efferent fibers (S2-4) is to mediate sexual response in the lower portion of the vagina.

The ilioinguinal and genitofemoral nerves originate from the upper part of the lumbar plexus of spinal nerves. Both nerves innervate the inguinal skin. In females, the genital branch of the genitofemoral nerve ends in the skin of the mons pubis and the ventral half of the labia majora. These nerves are exclusively sensory except for the genital branch of the genitofemoral nerve, which is responsible for the cremasteric reflex in men.
In view of these anatomical considerations, history-taking must focus on the external genital organs. The vulva and the vagina are the key organs for a proper neuropelveological diagnosis of chronic pelvic pain. Deep vaginal pain corresponds to irritation of the inferior hypogastric plexus and is therefore characterized by visceral pain accompanied by vegetative symptoms. The presence of anterior vulvodynia in combination with groin pain (which may or may not radiate to the thigh) suggests a pathology of the genitofemoral nerve (or lumbar plexus). Pudendal nerve pathology (Alcock's canal syndrome) is always combined with vulvodynia, perineal pain, and perianal pain. The combination of pudendal pain with non-gynecological pain, such as sciatica, gluteal pain, or low-back pain, suggests a pathology of the sacral plexus (sacral radiculopathy). Further pain that radiates to the anterior part of the thigh (lumbar dermatomes) generally corresponds to a spinal cord and/or spinal column disorder.

\section{Neuropelveological assessment of neuropathic pelvic pain}

\section{History}

Patients are asked a set of questions regarding potential causes (previous surgery, operative vaginal delivery, episiotomy, endometriosis, etc.), time of onset, duration, and severity of pain using a pain intensity scale (visual analog scale, VAS) of 0 (no pain) to 10 (worst imaginable pain) for standardized quantification of pain.

Risk factors for nerve damage Previous pelvic/abdominal surgeries or obstetric events are very important risk factors. All inguinal procedures such as surgical repair for inguinal hernia, low abdominal trocar or drainage incision, and Pfannenstiel incisions are associated with a risk of damage to the genitofemoral and/or ilioinguinal nerves. Laparoscopic and laparotomic approaches to the pelvic side wall or parametric tissues place patients at risk of damage to the somatic pelvic nerves, while central pelvic surgeries (hysterectomy and laparoscopic uterosacral nerve ablation (LUNA) $\rightarrow$ damage to the inferior hypogastric plexus, and presacral neurectomy $\rightarrow$ damage to the superior hypogastric plexus) expose patients to a risk of visceral pelvic pain. Perineal, proctological, and obstetrical interventions expose the pudendal nerve and its branches to a risk of surgical damage.

Pelvic interventions and thrombosis may also promote changes in pelvic vein circulation that may trigger the development of pelvic varicose veins, which are risk factors for vascular entrapment or sacral compartment syndrome. Because the constitution of pelvic and lower limb veins is similar, patients with varicose veins in the legs may also have a higher risk of pelvic varicose veins. 
History-taking should also focus on pathologies of the spinal cord, vertebral column, central nervous system (multiple sclerosis, Parkinson's disease, etc.), and peripheral nervous system (polyneuropathy).

Last but not least, the use of pain killers should be thoroughly documented.

Characteristics of pain-quality, location, and radiation Because visceral nerves extend through the pelvic organs like a spider web, visceral pelvic pain is usually described as vague, poorly localized, and dull pain, or as general malaise rather than real pain, which generally radiates to the lower back along midline. Visceral pain is very often associated with vegetative symptoms such as nausea, vomiting, pallor, diaphoresis, and tachycardia.

Somatic pelvic pain occurs due to somatic pelvic nerve damage and is classified as truncular (damage to the sciatic, obturator, femoral, or pudendal nerves) or radicular (damage to the lumbar or sacral plexus). Neuropathic pain causes numerous symptoms, ranging from allodynia, paresthesias, and sensations of electrical discharges to phantom sensations, which are even more confusing when no morphologic correlates are found. Hyperesthesia is usually the result of lesion causing nerve irritation, while hypoesthesia, anesthesia, and phantom pain generally develop after neurogenic nerve damage (axonal nerve damage). Somatic pain is well-described as localized pain with distal radiation in the corresponding dermatome(s) (Fig. 1). Generally, there is a trigger point at the level of irritation. Further symptoms may include increased sensitivity, electric shock-like sensations and/or stabbing, knife-like or aching pain, lump or foreign body sensation, twisting or pinching sensations, abnormal temperature sensations, constipation, pain and straining with bowel movements, straining or burning on urination, painful intercourse, and sexual dysfunction, including hyperarousal or decreased sensitivity.
Aggravating or relieving factors Pudendal neuralgia caused by compression of the pudendal nerve in Alcock's canal (Alcock's canal syndrome) typically induces severe pudendal nerve pain on sitting, which is relieved by standing, and absent when recumbent or when sitting on a toilet seat. Neuropathic pain induced by endometriosis of the pelvic nerves increases during menstruation and may decrease in response to gonadotropin-releasing hormone $(\mathrm{GnRH})$ analogues. In pelvic nerve irritation by vascular entrapment or compression (sacral compartment syndrome), pain is aggravated by all situations that induce an increase in pelvic venous pressure (prolonged standing or sitting, the Valsalva maneuver, etc.) or marked pulsation of the pelvic veins (tricuspid insufficiency, close anatomic relationship with arteries, etc.).

\section{Gynecological examination}

The clinical examination focuses on inspection of the genital organs (colposcopy), supported by vaginal culture, urinalysis, vaginal $\mathrm{pH}$ testing, Pap smear, and biopsy of abnormal vulvar areas. Recto-vaginal palpation not only focuses on the parametria and rectovaginal space, but also on the pudendal nerves and the low sacral nerve roots (the sacral nerve roots L5 and S1 are not accessible by vaginal or rectal palpation). Direct digital palpation of the pelvic nerves is the key to diagnosis of pelvic nerve dysfunction. The affected patients have exquisite tenderness when digital pressure is applied to the damaged nerve, typically producing Tinel's sign (sensation of tingling or "pins and needles") in the distal distribution of the nerve. The pudendal nerve is accessible to palpation a few millimeters dorsomedial to the sciatic spine, while the low sacral nerve roots are accessible at the sacral bone, a few centimeters left and right of midline (Fig. 1). Selective nerve block is crucial for confirmation of the diagnosis. Pudendal nerve block can be performed via a perineal approach with concomitant vaginal/rectal palpation,
Fig. 1 Neuropelveologic assessment of dermatomes and trigger points of the pelvic nerves. ( $L 5$ fifth lumbar root, $S$ sacral nerve root, $P N$ pudendal nerve, $S N$ sacral nerve roots, $G F N$ genitofemoral nerve)

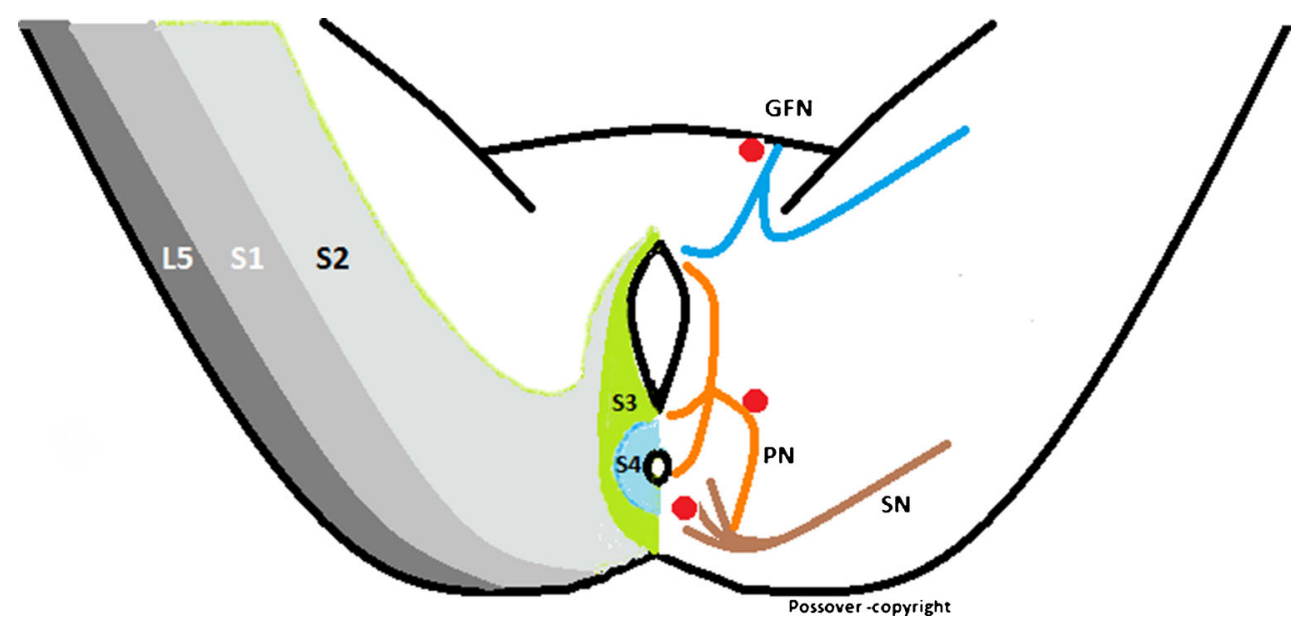


while sacral nerve root block can be achieved by epidural or dorsal transforaminal epidural injection.

\section{Neurologic examination}

A full neurological examination includes assessment of both the motor and sensory systems.

Muscle tone and power Ankle clonus is checked by placing the patient's leg turned outwards on the bed, moving the ankle joint a few times to relax it, and then sharply dorsiflexing it. Any further movement of the joint may suggest clonus. The patient is also assessed for possible motor deficits in hip adduction (L3/obturator nerve), knee extension (L1-L4/femoral nerve), ankle dorsiflexion (foot drop-L5), and ankle plantar flexion (S1).

Reflexes The patellar reflex corresponds to the L3 root, whereas ankle jerk corresponds to the $\mathrm{S} 1$ root.

Patient walking and Romberg's test When the patient is standing with the feet apart and the eyes closed, any swaying may be suggestive of posterior column pathology.

Sensation The patient is assessed for light touch and pin prick sensation in all lumbosacral dermatomes. Extrinsic lesions $(\rightarrow$ nerve irritation) do not cause any loss of sensation or feeling of numbness, while neurogenic nerve damage is accompanied by a loss of sensation or numbness in the corresponding nerve distribution.

Vibration Assessed using a sounding tuning fork placed on the medial malleolus.

Proprioception Assessed by up and down movement of the joint of the great toe ( $\rightarrow$ joint position sense).

Vaginal ultrasound

Vaginal ultrasound is not only used for assessment of organs and structures within the female pelvis, but also for evaluation of postvoid residual urine volume and bladder wall thickness. Pelvic varicose veins can also be identified directly via transvaginal Doppler ultrasound. Pelvic varicose veins is defined as the presence of dilated (diameter $\geq 7 \mathrm{~mm}$ ), tortuous vessels with reflux (presence of bidirectional flow during Valsalva's maneuver).

Renal ultrasound

Routine renal ultrasound may be recommended in all patients with pelvic endometriosis for prevention of silent ureteral stenosis, especially when deeply infiltrating endometriosis is suspected.

\section{Urodynamic testing}

Pathologies of sacral nerve roots S2-4 and/or of the pelvic splanchnic nerves induce neuropathic pain as well as lower urinary tract and intestinal dysfunctions. Such disorders may be evaluated by urodynamic testing (cystometry, uroflowmetry, and pressure flow studies), pelvic EMG recording, and video-urodynamic studies. When assessing bladder function, it is very important to differentiate between detrusor hypotonia, which indicates neurogenic damage to the pelvic nerves, and irritation of the pelvic nerves, which is associated with bladder hypersensitivity or overactive bladder (OAB). Urgency, the cardinal symptom of OAB, is defined as a sudden and compelling desire to pass urine that is difficult to defer, while bladder hypersensitivity is a urodynamic diagnosis characterized by an early desire to void without fear of leakage or pain that persists and becomes annoying to the patient. Bladder hypersensitivity may resemble irritation of the pelvic nerves in terms of an incontrollable desire to pass urine without leakage and without a rise in detrusor pressure during filling cystometry, but less so with pathologies of the bladder itself.

\section{Neurophysiological testing}

Some of the more commonly used tests include the pudendal nerve terminal motor latency test (PNTML), electromyography (EMG), and magnetic resonance neurography (MRN). EMG studies of the pudendal nerve, often touted as a diagnostic tool, are unreliable since they can be abnormal after vaginal delivery or vaginal hysterectomy. Moreover, they do not define the neurologic level of the pathology.

\section{Most frequent neuropathic pelvic pain}

Pudendal neuralgia —Alcock's canal syndrome

The diagnosis of pudendal neuralgia is reserved for patients with allodynia in the entire distribution of the pudendal nerve (vulvar, perineal and perianal area) that does not radiate to other lumbosacral dermatomes The pain is typically more severe when sitting, relieved by standing, and absent when recumbent or when sitting on a toilet seat. Various other symptoms may occur in some cases, for example urinary hesitancy (difficulty starting the flow of urine), frequency (frequent need to pass urine), urgency (sudden sensation to pass urine), constipation/painful bowel movements, reduced awareness of defecation (the process of passing bowel motions), sexual dysfunction including loss of libido. In neurogenic PN 
damage, loss of sensation or numbness selectively is usually combined with contralateral anal deviation due to homolateral perineal/perianal myoatrophia. Urodynamic testing can show bladder overactivity [2] but may also be normal. Urethral incontinence occurs only in bilateral neurogenic PN damage. By transrectal/vaginal palpation of the pudendal nerve at the sacrospinous ligament, patients have exquisite tenderness with a Tinel's sign in genitoanal area. There are numerous possible causes for pudendal neuropathy. Some of the possible causes are an inflammatory or autoimmune illness, frequently interpreted as infection. After iatrogenic nerve damage, which are frequent in obstetrics and gynecology pudendal neuralgia is common, with etiologies such as compression of the nerve through a postpartum hematoma, fibrosis of the ischiorectal fossa, stretching of the nerve during delivery, or surgical damage during transvaginal sacrospinous colpopexy [3]. Recent interventions using mesh material for sacrospinal fixation [4], sacrocolpopexy, or rectopexy may also expose patients to a risk of pudendal nerve damage [5].

\section{Genitofemoral neuropathy}

When the genitofemoral nerve is affected, the pain may be localized in the inguinal area and may radiate to the internal aspect of the thigh and to the genital area. Lesions of the genital branch of the genitofemoral nerve induce vulvodynia or pudendal pain located selectively in the anterior portion of the vulva (clitoris). Surgical access to the inguinal region (appendectomy, herniorraphia, introduction of lateral trocar for laparoscopy, etc.) exposes patients to a risk of injury to the genitofemoral nerve. Neurologic symptoms are then restricted to sensory changes located in the groin area, the ventral genital area (as in lesion of the ventral branch of the PN), and/or the internal aspect of the tight (lesion of the femoral branch) but never below the knee. Since the genital branch is only sensitive in females, neither bladder dysfunction nor urinary incontinence occurs. Inguinal nerve block with an anesthetic agent is the diagnostic method of choice.

\section{Sacral radiculopathies}

The incidence of sacral radiculopathies is widely underestimated due to the lack of awareness that such lesions exist, but also because of a lack of diagnosis, acceptance, declaration, and reporting, especially when such injuries occur secondary to surgical interventions. Pudendal neuralgia, on the other hand, is often over-diagnosed. In a series of 136 consecutive patients suffering from intractable pudendal pain, only 18 had true PN entrapment, while all of the others had a sacral radiculopathy [6].

Sacral radiculopathy combines "pelvic symptoms" such as pudendal pain, low abdominal pain, vulvodynia, dyspareunia/ apareunia, and coccygodynia with non-pelvic symptoms, including low-back-pain, gluteal pain, and sciatica. Massive lesions of the sacral nerve roots (plexopathy) are characterized by a loss of strength in hip extension, knee flexion, and dorsal plantar flexion of the foot. Because the vesical parasympathetic neurons (pelvic splanchnic nerves) are contained in sacral nerve roots S3-5, vesical symptoms are virtually constant. In extrinsic nerve irritation, bladder hypersensitivity is quasi systematic; true bladder overactivity may even be observed. Axonal nerve damage is characterized by loss of sensitivity or even numbness in the corresponding dermatomes, and ultrasound usually reveals postvoid residual urine; urodynamic testing confirms detrusor hypotonia or atonia as well as bladder hyposensitivity and increased bladder capacity.

Uterine myomas, ovarian processes, retroperitoneal vascular abnormalities (vascular entrapment), retroperitoneal fibrosis, and neurogenic tumors are frequent pelvic conditions that may induce pelvic neuropathies. However, the most common causes of sacral radiculopathy are surgical damage, deeply infiltrating endometriosis of the pelvic side wall, and nerve compression/entrapment by pelvic varicose veins [7]. Surgical nerve injuries occur due to coagulation, suturing, ischemia or cutting and induce disorders of sensation, pain, and dysfunction starting immediately after the procedure or within a few days. In contrast, nerve lesions caused by fibrotic tissue or vascular compression/entrapment usually require several months or years to develop. When diagnosing surgically induced sacral radiculopathies, correlation of clinical information with the surgical steps of the procedure permits precise anatomical localization of the neural lesion. This is necessary to adapt the treatment strategy accordingly. Perineal procedures may induce pathologies of the pudendal nerve and abdominal/laparoscopic procedures may affect the sacral nerve roots, while vaginal surgeries can induce both [5]. In a series of 92 consecutive patients with pelvic nerve damage secondary to surgery for pelvic organ prolapse (confirmed by laparoscopic exploration), the most frequent types of nerve damage were injuries to the right S2 nerve root incurred during laparoscopic rectopexy and laparoscopic colpopromontofixation, and injuries to the left S2 nerve root after vaginal uterosacral ligament suspension (McCall procedure). Vaginal mesh implantation for pelvic organ prolapse exposes patients to a risk of secondary nerve entrapment due to scar tissue development, especially when it involves new devices and techniques for blind needle-driving and minimal dissection. The reason is that, when any bleeding occurs, hematomas cannot drain and tend to dissect in retroperitoneal spaces, even in supralevator compartments, resulting in the formation of retroperitoneal fibrotic tissue.

When nerve injuries have occurred, laparoscopic exploration not only provides a tool for anatomic and functional exploration of the nerves, but also for effective neurosurgical treatment using techniques of nerve decompression or reconstruction. 
Most pelvic conditions responsible for compression or irritation of the sacral nerve roots can be treated well by laparoscopic surgery. Moreover, neurogenic lesions are accessible to laparoscopic surgery. The "LION procedure" for laparoscopic implantation of neuroprosthesis to the pelvic nerves enables symptomatic pain treatment by selective neuromodulation of damaged nerves [8]. Laparoscopy is therefore an essential and logical step in the management of pelvic nerve pathologies that should be performed as soon as possible, before the nerve damage becomes irreversible and before the pain chronification process has begun.

\section{Conclusions}

The main symptom of CPP is chronic pelvic pain, and the main objective of treatment is pain control. Chronic pelvic pain may be caused by gynecologic, dermatologic, or urological disorders as well as by central or peripheral neuropathic conditions. When performing the history and clinical examination, the clinician must consider the combined gynecological, urological, and neurological aspects of pain. For proper diagnosis of CPP, the best way of thinking is to take a neuropelveological approach, which does not initially focus on diagnosing possible pelvic pathologies, but rather on identifying the neurologic pathways responsible for transmission of the pain signals to the central nervous system. Once these have been identified, the next step is to determine the level of the lesion (below, in, or above the pelvis). Determination of the etiology is the last step of neuropelveological diagnosis.

Because neuropelveologic assessment is based on the collection of many small clinical details gathered during careful history-taking and clinical examination, it takes time. Nevertheless, this is simple clinical methodology. An exact neuropelveological diagnosis can be established in most patients suffering from intractable CPP, making it possible to optimally adapt the treatment. Targeted therapeutic options include local injections of local anesthetics, corticoids or botulinum toxin A (Botox), laparoscopic techniques for nerve decompression, neurolysis or neuromodulation (LION procedure), and neurosurgical/orthopedic spinal procedures.
Medical pain treatment is a real option for pain control, and all patients undergoing laparoscopic procedures involving the pelvic nerves must receive such pain management treatment for a certain period. However, if etiological treatment is available, it will have priority over symptomatic treatment. The LION procedure for the implantation of electrodes to pelvic nerves is an innovative treatment option which is indicated only in neurogenic situations with combined pelvic pain and dysfunction. In the past, most patients were subjected to multiple examinations by multiple physicians as well as to strong medications that often caused side effects and reduced quality of life. With the new treatments available today, such problems are now avoidable.

Disclosure The authors declare that they have no conflict of interest.

\section{References}

1. Reitz A, Schmid DM, Curt A, Knapp PA, Schurch B (2003) Afferent fibers of the pudendal nerve modulate sympathetic neurons controlling the bladder neck. Neurourol Urodyn 22(6):597-01

2. Virseda Chamorro M, Salinas-Casdo J, Zarza-Lucianez D, MendezRubio S, Pelaquim H, Esteban-Fuertes M (2012) Participation of the pudendal innervation in the detrusor overactivity of the detrusor and in the overactive bladder syndrome. Actas Urol Esp 36(1):37-41

3. Verdeja AM, Elkins TE, Odoi A, Gasser R, Lamoutte C (1995) Transvagnal sacropsinous colpopexy: anatomic landmarks to be aware of to minimize complications. Am J Obstet Gynecol 173:1468-1469

4. Debodinance P, Amblard J, Fatton B, Cosson M, Jacquetin B (2007) The prosthetic kits in the prolapsed surgery: is it a gadget? J Gynecol Obstet Biol Reprod 36(3):267-275

5. Possover M, Lemos N (2011) Risks, symptoms, and management of pelvic nerve damage secondary to surgery for pelvic organ prolapse: a report of 95 cases. Int Urogynecol J 22(12):1485-1490

6. Possover M (2009) Laparoscopic management of endopelvic etiologies of pudendal pain in 134 consecutive patients. J Urol 181:17321736

7. Possover M, Schneider T, Henle KP (2011) Laparoscopic therapy of endometriosis and vascular entrapment of sacral plexus. Fertil Steril 95:756-758

8. Possover M (2010) New surgical evolutions in management of sacral radiculopathies. Surg Technol Int 19:123-128 